\title{
Structural Raman Enhancement in Graphite Nano-Discs
}

J. F. Cardenas, ${ }^{1,2}$ D. Chakarov, ${ }^{3}$ B. Kasemo ${ }^{3}$

${ }^{1}$ Department of Chemistry, University of Oslo,

Sem Sælands vei 26, 0371 Oslo, Norge.

${ }^{2}$ Present affiliation:

Departamento de fisica, Escuela Politecnica Nacional,

Ladron de Guevara E11-253,

EC 170109, Quito

Ecuador

${ }^{3}$ Department of physics, Chalmers University of Technology,

SE-412 96, Gothenburg, Sweden. 


\begin{abstract}
Raman scattering in disc-shaped graphite nanostructures, etched out of bulk HOPG, are investigated using an excitation wavelength of $532 \mathrm{~nm}$ at different laser power. The G-band is fitted using two Lorentzian functions, $\mathrm{G}_{\mathrm{L}}$ and $\mathrm{G}_{\mathrm{H}}$. The difference of Raman shift between the two Lorentzian functions increase with laser power as a consequence of selective absorption and heating of the discs. Further, the G-band from the nanostructured HOPG reveal a Raman enhancement (RE) of $\sim 2.2$ and $\sim 1.5$ for the components associated with the discs $\left(G_{L}\right)$ and the supporting substrate $\left(G_{H}\right)$, respectively. The quantitative agreement between the experimental results and performed finite difference time domain calculations make possible to conclude that electromagnetic energy penetrates considerably into the discs from the circular periphery probably due to multiple scattering. In addition, the dependence of RE of the $G_{L}$ component on the laser power is attributed to a temperature dependent electron-phonon coupling.
\end{abstract}




\section{Introduction}

Light scattering properties of nanoparticles depends on their electronic structure, size and geometrical shape. ${ }^{1}$ For a small particle diameter (D) to wavelength $(\lambda)$ ratios $(D / \lambda<<1)$, light scattering by metallic spheres can be described as emission of light from an resonant dipole (Rayleigh approximation). In this limit the dipole exhibits a resonance when the wavelength of light approaches the plasma frequency of the metallic particle, i.e. the resonance frequency of the dipole depends on the shape but not on the size. ${ }^{2}$ The interference of incoming and emitted light results in enhanced electric field in the vicinity of the metallic nanoparticles (the nearfield effect) and is, for example, responsible for surface enhanced Raman scattering (SERS). ${ }^{3}$ The field effect of SERS on molecules attached to metallic nanoparticles

accounts for an enhancement factor of up to $10^{10},{ }^{4}$ while the chemical effect adds to the enhancement by factor of about $10^{5} .{ }^{5}$ On the other hand, when light is incident on dielectric nano- and microparticles, the light scattering characteristics show resonance features when the frequency of the incident field coincides with the electromagnetic eigenmodes of the particle $^{.6,7}$ Such resonant excitation leads to sharp features in the photoluminescence (CdS), ${ }^{8}$ and Raman ${ }^{9,10}$ scattering spectra (with a Raman enhancement,RE, of $\sim 10-1000$ ). In this case the resonance conditions depend on the shape and size of particles.Light scattering of graphite particles with a radius of $\sim 100 \mathrm{~nm}$ are in the intermediate regime between Rayleigh and Mie scattering for $\mathrm{l}=532 \mathrm{~nm} .{ }^{11}$ Graphite is a semimetal without bandgap and very low electron density around the Fermi level. These two facts, size and electron structure, and places the system investigated here in a special, intermediate position in respect to the scattering regime. In contrast to dielectric materials like silicon where the size of the nanoparticle is much smaller than the inverse of the absorption coefficient, the support of eigenmodes is not expected to be significant in graphite structures. Here we argue that modest RE from disc-shaped nanostructures on graphite surfaces is due to multiple scattering of light between the discs.

\section{Experimental and simulation methods}

Disc-shaped graphite nanostructures are etched out of bulk HOPG sample using hole-mask colloidal lithography (HCL) in combination with oxygen reactive ion etching (ORIE). The method and its supporting procedures have been described thoroughly elsewhere. ${ }^{12}$ In short, HCL is used to prepare arrays of gold discs with well-defined diameters on the surface of an HOPG sample (grade ZYB, GE Advanced ceramics). This technique utilizes self-assembly of colloidal spheres to produce uniform arrays of well-separated nanostructures but with no long-range order. The gold disc arrays 
are subsequently used as etch masks in the ORIE process, which in a controlled way removes carbon atoms from the HOPG surface not protected by the gold discs. Hence, the gold disc pattern is transferred into the HOPG, resulting in slightly tapered HOPG discs etched out of the bulk HOPG. Finally, the gold discs are removed in a wet-etch process using a potassium-iodine solution (Gold etch 22196, Sunchem electrograde products) and cleaned in acetone and deionized water. In addition to the nanostructured samples we also investigate the reference samples, a pristine HOPG. The nanostructured samples will be referred to as HOPG147 and HOPG217 (number indicates diameter of discs in nm) and the pristine will be referred to as HOPGp.

The size and distribution of the nanodiscs on the HOPG-surfaces are characterized by SEM (SEM JEOL- JSM630 1F) and AFM (DI-dimension 3000 SPM). Diameters, heights and surface coverage of the discs are evaluated from the obtained images using ImageJ and the scanning probe image processor (SPIP, version 4.5.7, Image Metrology Inc., Denmark) software, respectively. Raman scattering measurements are performed using a Jobin Yvon T64000 spectrometer. The measurements are done through an objective (x50 with a numerical aperture of 0.5), using backscattered light from a spot of about $4 \mu \mathrm{m}^{2}$, excited by a laser $(\lambda=532 \mathrm{~nm})$ beam of different intensities, $\mathrm{I}_{\mathrm{L}},(9.7$ to $46.7 \mathrm{~mW})$. The measurements are performed, first in the anti-Stokes wavenumber regime $\left(-1750\right.$ to $\left.-1250 \mathrm{~cm}^{-1}\right)$ and then, for the same sample location, in the Stokes regime $\left(1250\right.$ to $\left.1750 \mathrm{~cm}^{-1}\right)$.

The fitting procedure, which is of high importance for the results obtained here, was performed in the following way. The data for the HOPG-RIE and the HOPG-ref samples at different laser intensities were each fitted with a single Lorentzian, obtaining reference values for the width and frequency dependence on laser intensity. When fitting the data for the HOPG147 and HOPG217 samples, two Lorentzian functions, denoted as $G_{H}$ and $G_{L}$ were used, where the width and Raman shift of the $\mathrm{G}_{\mathrm{H}}$ components were assumed to have the same (within a small tolerance, i.e., Raman shift above $1581 \mathrm{~cm}^{-1}$ at low and $1580 \mathrm{~cm}^{-1}$ at the highest laser intensities) values as obtained during the fitting of the data of the HOPG-RIE sample. Note that, the $\mathrm{G}_{\mathrm{H}}$ and the $\mathrm{G}_{\mathrm{L}}$ components are associated with the substrate and the discs, respectively. The widths and Raman shifts of the $G_{L}$ component was allowed to vary without restrictions during the fitting procedure. It is obvious that at low laser intensities the $G_{H}$ and the $G_{L}$ component will overlap and thus a separation of the components is not possible. With increasing laser intensity, the discs selectively heats compared to the 
substrate and thus the separation of the two components becomes possible. ${ }^{13}$ In the calculation of RE the integral area of each component rather than its amplitude represents the peak intensity.

In order to simulate the incidence of plane waves onto graphite disc/layer structure we performed finite difference time domain calculations using MEEP ${ }^{14}$. The simulations were performed for a structure with a disc diameter and height of $170 \mathrm{~nm}$ and $110 \mathrm{~nm}$, respectively, on top of a graphite layer with a thickness of $100 \mathrm{~nm}$. The thickness of the supporting layer was sufficiently large to absorb most of the light and, most importantly, suppresses significantly the reflection on the backside of the layer. While the simulated structure is not exactly the one used for the Raman scattering experiments the simulation results and conclusions remain relevant when comparing with experiments. Periodic boundary conditions were used for sides of the simulation volume parallel to the direction of the generated plane waves. Perfect matchinglayers were incorporated on the sides perpendicular to the incidence direction of the generated plane waves. The resolution of the grid was $2 \mathrm{~nm}$ in all directions. The anisotropic material properties of graphite were taken into account. Thus, the complex dielectric constants of graphite $\varepsilon^{\perp}$ and $\varepsilon^{\|}$describe induced polarizability when the electric field is oriented perpendicular and parallel to the c-axis of graphite, respectively. ${ }^{15}$

\section{Results and Discussion}

In Fig. 1 the G-band, from the Raman scattering of the HOPG147 sample, irradiated with $\mathrm{I}_{\mathrm{L}} \sim 44 \mathrm{~mW}$, is fitted using two Lorentzian components, $\mathrm{G}_{\mathrm{H}}$ and $\mathrm{G}_{\mathrm{L}}$ centered at 1580 and $1571 \mathrm{~cm}^{-1}$, respectively. Note that the dependence of the Raman shift (shown in the inset of Fig. 1) of the $G_{L}$ component on laser power implies a temperature increase of the discs with increasing $\mathrm{I}_{\mathrm{L}}$, since Raman shift is inversely proportional to temperature.13 (Similarly???), in Fig. 2 the dependence of the intensity of the $G_{L}$ and the $G_{H}$ components on $I_{L}$ for the HOPG147 and the HOPG217 sample are shown. The $G_{H}$ intensity versus $I_{L}$ reveals a linear relation for both samples. !!! Show this on the Fig.!!!, which cross the origin (within an uncertainty of $1 \mathrm{mw}$ ) in contrast to the dependence of the $\mathrm{G}_{\mathrm{L}}$ component on $\mathrm{I}_{\mathrm{L}}$. The extrapolation of the dependence of the intensity of $G_{L}$ on $I_{L}$ has an intersection at a power level of $\sim 8 \mathrm{~mW}$ (for both the HOPG147 and the HOPG217 sample). This implies that the dependence of the intensity of the $G_{L}$ component on $I_{L}$ varies with $I_{L}$. 
Further, we incorporated in the analysis the dependence of the G-band intensity on $\mathrm{I}_{\mathrm{L}}$ for an unstructured HOPG (reference) sample, and found that the $\mathrm{G}_{\mathrm{L}}$ and $\mathrm{G}_{\mathrm{H}}$ components for both samples (HOPG147 and HOPG217) are enhanced compared to the reference sample. Note, that the fractional areas of 0.2 and 0.8 for the $G_{L}$ and $G_{H}$ components, respectively, are taken into account in Fig. 2. The enhancement of the $\mathrm{G}_{\mathrm{H}}$ component for the HOPG147 and the HOPG217 samples are 1.70 and 1.30, respectively. Moreover, the enhancement of the $\mathrm{G}_{\mathrm{L}}$ component for the HOPG147 and the HOPG217 samples are 2.39 and 2.12, respectively. Thus, the relative increase in enhancement is higher for the $\mathrm{G}_{\mathrm{H}}$ component compared to the $\mathrm{G}_{\mathrm{L}}$ component when the characteristic disc diameter and inter-disc distance are decreased. Hence, using selective laser induced heating we are able to obtain position specific information on the electric fields and on absorption (heating) in a similar approach as this was demonstrated previously for porous silicon structures. ${ }^{16}$

We assume that light scattering by the discs results in a multi scattering cascade, where light is confined between the discs, ie. propagates parallel to and in the vicinity of the HOPG surface. Eventually light will be backscattered from or absorbed on the sides of the discs and on the HOPG surface between the discs. This will give an additional contribution to the Raman scattering within the discs compared to the situation when the light only propagated perpendicular to the HOPG surface. Moreover the bouncing of light between the discs will also increase the field density of the HOPG between the discs, compared to the case when it propagates (only) perpendicular to the planar HOPG surface.

The numerical simulation of a PW onto a graphite disc/layer structure with diameter $110 \mathrm{~nm}$ (Fig. 3) results in polarization of the disc with two sides (opposite to each other where the symmetry axis is parallel to the electric field, E, and Y-axis) having the highest energy density. Note that as an effect of the anisotropic $\varepsilon$-value the energy density,

$u_{e}=(\varepsilon-1) \mathrm{E}^{2}$

is attenuated exponentially only along the direction of the c-axis, but not perpendicula to the c-axis. This suggests that PWs with an incidence direction parallel to the HOPG surface, due to multiple scattering, is enhancing the field intensity within the disc. In addition, the non-exponential dependence of $u_{e}$ with the radial distance from the center of 
the disc is indicative for possible support of eigenmodes by the discs.

Raman scattering is proportional to the square of the induced polarization and thus RE can be defined as the ratio of $u_{e}$ within the graphite disc to that of a planar reference HOPG substrate. ${ }^{17}$ The calculated ratio of the integrated $u_{e}$ of the discs is about 6.9 times larger than in the reference substrate for a periodic array of discs (Fig. 3). Note, in the case of graphite it is important to take into account the attenuation of the Raman scattered light since signal is proportional the convolution of $u_{e}$ with an exponential depth-dependent function. Thus after convoluting the depth distribution of $u_{e}$ (for the structured graphite and the reference structure) with an exponential depth dependent function the ratio is 3.4, in rough agreement with experiments. A similar analysis for an isolated Si-sphere with a diameter of $112 \mathrm{~nm}$ yields an enhancement of 20 in excellent agreement with experimental results. ${ }^{9}$ While the size of the graphite disc and the Si sphere are of about the same size, in the latter particle the electronic properties enable the support of structural eigenmodes in contrast to graphite, which is too dissipative to support such modes.

Within a dielectric nanoparticle with $\mathrm{D}<<\lambda$ the internal field is diminished compared to the incident field, $\mathrm{E}_{0}$, due to the depolarization effects. For a small sphere the field inside is ${ }^{18}$

$$
E_{x}=\frac{E_{0}}{\left(1+N_{x}\left(\epsilon_{r}-1\right)\right)}
$$

where $\mathrm{N}_{\mathrm{x}}$ is the depolarization factor, which is $1 / 3$ for a sphere and nearly so for a cylinder of this shape. ${ }^{19}$ Thus, when $\mathrm{D}<<\lambda$, the intensity of Raman scattering is predicted to be proportional to (excluding retardation effects): ${ }^{20}$

$$
I_{R} \propto E^{2} \frac{\left(\epsilon_{r}-1\right)}{\left(1+N_{x}\left(\epsilon_{r}-1\right)\right)^{2}}
$$

Note, the dependence on $u_{e}=(\varepsilon-1) \mathrm{E}^{2}$. Thus, compared to a planar substrate, the Rayleigh approximation predicts a decrease in intensity for an isolated nanoparticle that do not support eigenmodes, contrary to what is observed. Thus, 
when the size of the disc is in the region between the typical for Rayleigh and Mie scattering, eq. 3 above indicates that the observed RE is a consequence of the effect of multiple scattering within the space between the particles. Note that while the FDTD simulation accounts for a single disc, the simulation implicitly accounts for the co-existence of a periodic lattice of an infinite amount of identical discs next to each other. Thus, the enhancement observed in this work is of a different form to those observed in metallic and dielectric particles and is attributed to multiple scattering between the discs on the surface of the HOPG substrate. Note also, that for a constant surface coverage, it is expected that multiple scattering enhancement of the energy density increases with decreasing diameter of the discs due to a higher probability to undergo additional scattering events (to be confirmed in future work). However, due to the boundary conditions used here this prediction may be difficult to verify to inter-disc distance effects.

At low laser power even the limited cooling capacity of the substrate is sufficient to maintain the temperature of the discs equilibrated with the substrate For sufficient high laser power, a non-linear temperature dependence on laser power is predicted by theory ${ }^{21}$, and has been confirmed experimentally. ${ }^{22,}{ }^{23}$ Thus, for sufficiently high laser power the substrate cooling is not capable of maintaining a low temperature of the discs and a rapid rise in temperature is expected for the discs, which results in a non-linear dependence (Fig. 2 for the $G_{L}$ component) of the temperature of the discs on laser power. Note that the extrapolation of the dependencies of the Raman shift on $I_{L}$ for the $G_{L}$ and the $G_{H}$ component intercept at $\sim 5 \mathrm{~mW}$, which imply a non-linear Raman shift ( and temperature) of the $\mathrm{G}_{\mathrm{L}}$ component (disc) with laser power in an intermediate power regime! Further, since the graphite thermal conductivity (along the c-axis) drops quickly above $400 \mathrm{~K}$, from about $100-105 \mathrm{~W} / \mathrm{mK}$ at $400 \mathrm{~K}$ to $65 \mathrm{~W} / \mathrm{mK}$ at $950 \mathrm{~K}$, a turning point in the heat transport flow versus absorbed energy in the discs is expected at about $400 \mathrm{~K}$ to $500 \mathrm{~K} .{ }^{24}$ This explains why the temperature dependence on $\mathrm{I}_{\mathrm{L}}$ is non-linear.

Below we argue why the Raman cross-section increases with laser power (or in this case temperature) for nanostructured graphite in contrast to a HOPG surface that is not structured. ${ }^{25}$ The refraction index and extinction coefficient of graphite varies slightly with temperature (in the visible range by $1.5 \%$ between $25 \mathrm{C}$ and $600 \mathrm{C})^{26}$ and the graphite reflection coefficient (at $1064 \mathrm{~nm}$ ) decreases from 21 to 0.18 between 300 and 1300K. ${ }^{27}$ Hence, it is expected that a slight refraction index contrast will be induced between discs and substrate, when the discs are selectively heated 
by a laser beam. Due to the anisotropic thermal conduction properties of graphite this may result in increasing light absorption and Raman scattering with $\mathrm{I}_{\mathrm{L}}$. An additional reason for why RE is non-linear could be found in the fact that the Stokes intensity depends on $-\mathrm{n}_{\mathrm{BE}}$ (the negative of the Bose-Einstein distribution law), ${ }^{28}$ which accounts for an intensity increase of $10 \%$ between $300 \mathrm{~K}$ and $1000 \mathrm{~K}$. However, these two effects can be neglected.

The most important effect for the increase of Raman cross-section with temperature is related to the electron-phonon coupling (EPC). Raman scattering cross-section in crystals increases with the EPC magnitude. ${ }^{29}$ Moreover, Raman band linewidths depends on the anharmonicity of the vibration potentials and EPC, and in well ordered graphite, the anharmonic contribution is negligible between 0 and $900 \mathrm{~K}$ (i.e. the linewidth is constant with temperature). ${ }^{30}$ Thus, in graphite the linewidth depends only on the EPC. Hence, an increasing linewidth (and thus an increasing Raman crosssection) for a well graphitized structure implies an increasing EPC, which corroborates well with the observations: the linewidth of the $\mathrm{G}_{\mathrm{L}}\left(\mathrm{G}_{\mathrm{H}}\right)$ component increases by $\sim 60 \%(\sim 0 \%)$ between 0 and $44 \mathrm{~mW}$ (to be published in future work)! The interesting question why the EPC depends on temperature for nanostructured graphite but not for bulk graphite remains unanswered. We should point out that $\varepsilon$ depends on particle size (and temperature) and the absolute value of $\varepsilon^{\|}$ is highly uncertain (important for the multiscattering enhancement phenomena). ${ }^{15}$

\section{Conclusions}

The mechanism responsible for the Raman enhancement (RE) of the G-band of nanostructured (disc shaped) HOPG surfaces is investigated. RE factors for the nano-discs and the supporting substrate of $\sim 2.2$ and $\sim 1.5$, respectively, are observed for disc diameters between 100 and $200 \mathrm{~nm}$. FDTD calculations account for this magnitude and reveal an electromagnetic energy density distribution within the discs that do not decay exponentially, which indicate an effect of structural eigenmodes and multiscattering. Moreover, the RE of the discs depends on the laser power (and consequently on temperature), which is attributed to a temperature dependence of the electron-phonon coupling for nanostructured graphite. 


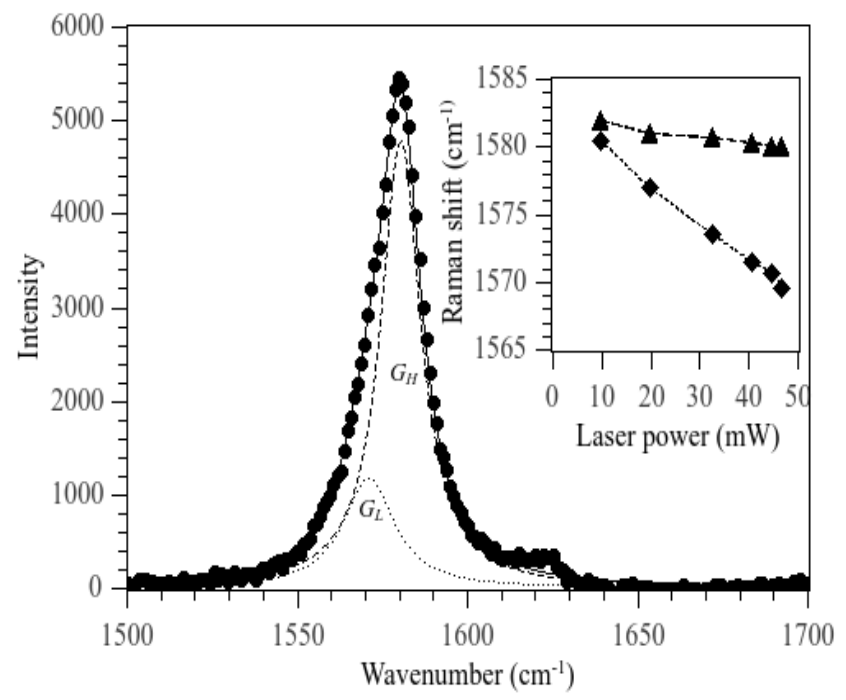

Fig. 1 The G-band of disc-shaped graphite nano structures, fitted using two Lorentzian functions ( $G_{H}$ and the $G_{L}$ components), from the HOPG147 sample at a laser power of $44 \mathrm{~mW}$. The inset show the Raman shift of the $G_{H}$ and the $G_{L}$ components as a function of laser power. 


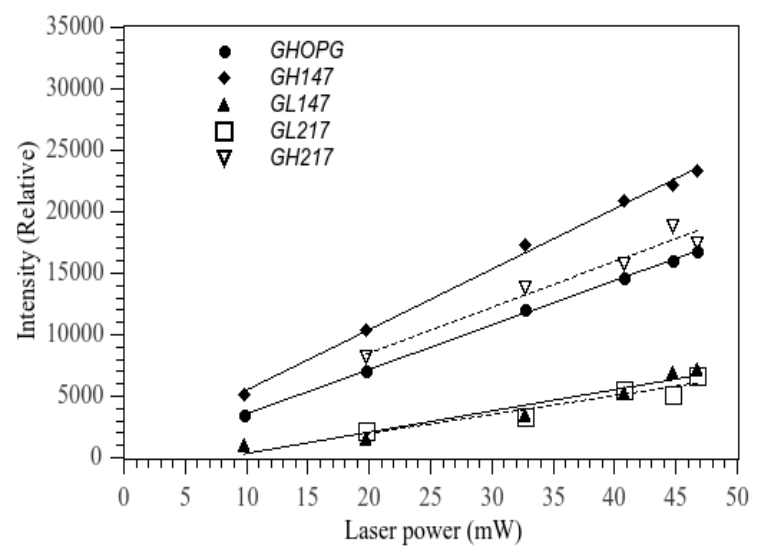

Fig. 2 The Raman scattering intensity of the G-band from the reference HOPG and the $\mathrm{G}_{\mathrm{H}}$ and the $\mathrm{G}_{\mathrm{L}}$ components from the HOPG147 and the HOPG217 samples. 


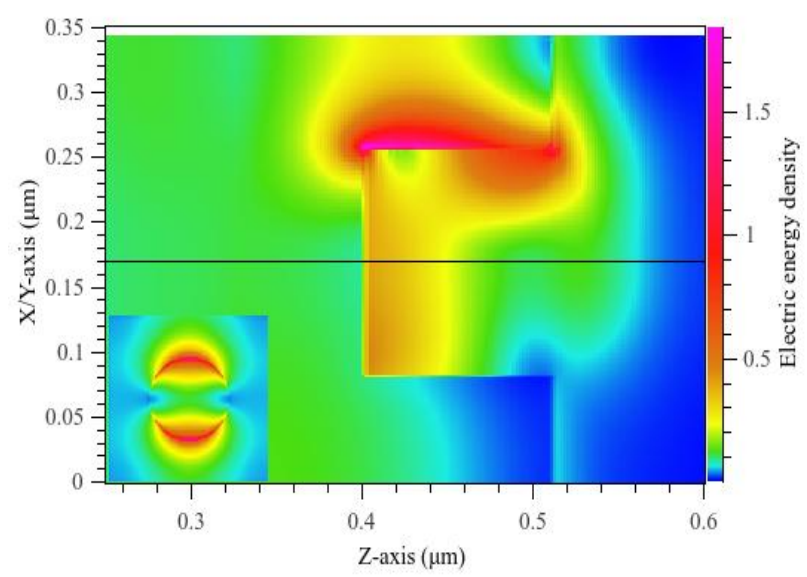

Fig. 3 Energy density distribution for an FDTD simulation of a plane wave incident on a graphite disc/substrate structure. Note the upper (Y-axis) and lower (X-axis) half of the large figure reveal the cross-section parallel and perpendicular to the electric field, respectively. The inset shows the cross-section perpendicular to the incidence direction at the interface between the disc and the substrate. The calculation is for $170 \mathrm{~nm}$ disc diameter .

\section{Acknowledgement}

J. F. Cardenas acknowledge financial support from the Norweigian research council. 
[1]Bohren, C. F. and Huffman, D. R., [Absorption and Scattering of Light by Small Particles], Wiley-VCH Verlag GmbH, Weinheim, ch.3 (1998). doi:10.1002/9783527618156.ch3

[2]Kelly, K. L., Coronado, E., Zhao, L. L. \& Schatz, G. C. “The Optical Properties of Metal Nanoparticles: The Influence of Size, Shape, and Dielectric Environment”, J. Phys. Chem. B 107, 668-677 (2003).

[3]Kerker, M., Wang, D.-S. \& Chew, H., “Surface enhanced Raman scattering (SERS) by molecules adsorbed at spherical particles”, Appl. Opt. 19, 3373 (1980).

[4]Ru, E. C. L. \& Etchegoin, P. G., “Single-Molecule Surface-Enhanced Raman Spectroscopy”, Annual Rev. Phys. Chem. 63, 65-87 (2012).

[5]Nie, S. \& Emory, S. R., "Probing Single Molecules and Single Nanoparticles by Surface-Enhanced Raman Scattering”, Science 275, 1102-1106 (1997).

[6]Chew, H. \& Wang, D.-S., “Double Resonance in Fluorescent and Raman Scattering by Molecules in Small Particles”, Phys. Rev. Lett. 49, 490-492 (1982).

[7]Lange, S. \& Schweiger, G., “Structural resonances in the total Raman- and fluorescence-scattering cross section: concentrationprofile dependence”, J. Opt. Soc. Amer. B 13, 1864 (1996).

[8]Wang, Y., “Local field effect in small semiconductor clusters and particles”, J. Phys. Chem. 95, 1119-1124 (1991).

[9]Murphy, D. V. \& Brueck, S. R. J., “Enhanced Raman scattering from silicon microstructures”, Opt. Lett. 8, 494-496 (1983).

[10]Cao, L., Nabet, B. \& Spanier, J. E., “Enhanced Raman Scattering from Individual Semiconductor Nanocones and Nanowires”, Phys. Rev. Lett. 96, 157402 (2006).

[11]Hong, S.-H. \& Winter, J., “Size dependence of optical properties and internal structure of plasma grown carbonaceous nanoparticles studied by in situ Rayleigh-Mie scattering ellipsometry”, J. Appl. Phys. 100, 064303 (2006).

[12]Fredriksson, H., Chakarov, D. \& Kasemo, B., "Patterning of highly oriented pyrolytic graphite and glassy carbon surfaces by nanolithography and oxygen plasma etching”, Carbon 47, 1335-1342 (2009).

[13]Cardenas, J. F., Fredriksson, H., Kasemo, B. \& Chakarov, D. "Light induced selective heating of nanostructured pyrolitic graphite surfaces investigated by Raman scattering”, Chem. Phys. 415, 232-236 (2013).

[14]Oskooi, A. F., Roundy, D., Ibanescu, M., Bermel, P., Joannopoulos, J. D. \& Johnson, S. G., "Meep: A flexible free-software package for electromagnetic simulations by the FDTD method”, Comp. Phys. Comm. 181, 687-702 (2010). 
[15]Draine, B. T. and Lee, H. M., “optical properties of interstellar graphite and silicate grains”, Astrophys. J. 285, 89-108 (1984).

[16]Deb, S. K., Mathur, N., Roy, A. P., Banerjee, S. \& Sardesai, A., "Raman scattering study of microstructure of n-type porous silicon”, Solid State Comm. 101, 283-287 (1997).

[17] Long, D. A. [Raman Spectroscopy], McGraw-Hill, New York, p.63 (1977).

[18] Jackson, J. D. [Classical elecrodynamics], Wiley, Hoboken, p.158 (1999).

[19]Venermo, J. \& Sihvola, A., “ Dielectric polarizability of circular cylinder”, J. Electrostat. 63, 101-117 (2005).

[20]Ndong, G., Picardi, G., Chaigneau, M. \& Ossikovski, R., “Retardation assisted enhanced Raman scattering from silicon nanostripes in the visible range”, Nanotech. 24, 035705 (2013).

[21]Lax, M., “Temperature rise induced by a laser beam II. The nonlinear case”, Appl. Phys. Lett. 33, 786-788 (1978).

[22]Lo, H. W. \& Compaan, A., “Raman measurements of temperature during cw laser heating of silicon” J. Appl. Phys. 51, 15651568 (1980).

[23]Abbott, D., Davis, B., Gonzalez, B., Hernandez, A. \& Eshraghian, K., “Modelling of low power cw laser beam heating effects on A GaAs substrate”, Solid-State Electron. 42, 809-816 (1998).

[24]Uher, C. [Thermal Conductivity of Pure Metals and Alloys], Springer, Berlin-Heidelberg, 430-439 (1991).

[25]Atashbar, M. Z. \& Singamaneni, S., “Comparative studies of temperature dependence of G-band peak in single walled carbon nanotube and highly oriented pyrolytic graphite”, Appl. Phys. Lett. 86, 123112 (2005).

[26]Stagg, B. J. \& Charalampopoulos, T. T., "Refractive indices of pyrolytic graphite, amorphous carbon, and flame soot in the temperature range $25^{\circ}$ to $600^{\circ} \mathrm{C}$, Combustion and Flame 94, 381-396 (1993).

[27]Bulgakova, N. M. \& Bulgakov, A. V., "Pulsed laser ablation of solids: transition from normal vaporization to phase explosion”, Appl. Phys. A 73, 199-208 (2001).

[28]Long, D. A. [Raman Spectroscopy], McGraw-Hill, New York, p.82 (1977).

[29]Loudon, R., “Theory of the First-Order Raman Effect in Crystals”, Proc. Roy. Soc. London A: Math., Phys. Eng. Sci. 275, 218232 (1963).

[30]Lazzeri, M., Piscanec, S., Mauri, F., Ferrari, A. C. \& Robertson, J., "Phonon linewidths and electron-phonon coupling in graphite and nanotubes”, Phys. Rev. B 73, 155426 (2006). 